\title{
THE EMBEDDING OF TWO-SPHERES IN THE FOUR-SPHERE
}

\author{
BY \\ HERMAN GLUCK( $\left.{ }^{1}\right)$
}

We consider the question of reducing the homeomorphism problem for pairs of topological spaces to the homeomorphism problem for single spaces. Of particular interest is the strength of the invariant $X-A$ of the pair $(X, A)$. In the first chapter we discuss the problem in its general setting. In the second chapter we consider the embedding of $n$-spheres in the $n+2$ sphere, and in particular the case $n=2$. Instead of the complement $S^{n+2}-S^{n}$, we use the exterior of $S^{n}$ in $S^{n+2}$ (i.e., the complement of an open regular neighborhood of $S^{n}$ ). We prove that there are at most two nonequivalent locally flat embeddings of $S^{2}$ in $S^{4}$ with homeomorphic exteriors. In certain classical cases, the exterior is a complete invariant for the embedding. In the third chapter we consider the structure of embeddings in more detail. The main result is that any locally flat orientable surface in four-space is the boundary of an orientable three-manifold in four-space. In the fourth and final chapter, we consider the embedding of $n$-spheres in the $n+2$ sphere for values of $n$ other than 2. The situation for $n>2$ resembles that for $n=2$, while the case $n=1$ stands apart from the rest.

\section{The GENERAL PROBLEM}

1. Introduction. One of the central problems of topology is that of discovering techniques for deciding whether two given spaces, $X$ and $X^{\prime}$, are homeomorphic. Related to this is the homeomorphism problem for pairs of spaces, $(X, A)$ and $\left(X^{\prime}, A^{\prime}\right)$, with $A \subset X$ and $A^{\prime} \subset X^{\prime}$. In this case the two pairs are said to be homeomorphic if there exists a homeomorphism $h$ of $X$ onto $X^{\prime}$ such that $h(A)=A^{\prime}$. It is customary to assume that the problem for single spaces "precedes" that for pairs of spaces, and hence to begin the second problem with the assumption that $X$ is homeomorphic to $X^{\prime}$ and $A$ to $A^{\prime}$. In this sense, the second problem really asks in what ways $A$ can be embedded in $X$. We refer to this as the knotting problem for $A$ in $X$. If $X$ and $A$ are manifolds, and the allowable embeddings restricted to some nice class, then there seems to be hope for reducing the knotting problem for $A$ in $X$

Received by the editors July 5,1961 .

(1) The author is a National Science Foundation Predoctoral Fellow, and wishes to thank Professor Ralph Fox for his help in the preparation of this paper, which is a condensation of the author's doctoral thesis. Thanks are also due the referee for many helpful suggestions. 
to a homeomorphism problem(s) for some nice class of spaces $\left({ }^{2}\right)$.

Henceforth, whenever we discuss the homeomorphism of $(X, A)$ with $\left(X^{\prime}, A^{\prime}\right)$ we shall always be assuming that $X$ is homeomorphic to $X^{\prime}$ and $A$ to $A^{\prime}$. The most immediate necessary condition for the homeomorphism of these pairs is the homeomorphism of $X-A$ with $X^{\prime}-A^{\prime}$. When $A$ is a point and $X$ a closed arc, this condition is of course sufficient. It is also sufficient when $A$ is a simple closed curve and $X$ is a closed two-dimensional separable Hausdorff manifold. The condition is, however, no longer sufficient when $A$ is a pair of disjoint simple closed curves and $X$ is Euclidean three-space, even though we may restrict the embeddings to be semi-linear (see [1]). Once we permit the embeddings of $A$ in $X$ to be wild, $X-A$ becomes a hopelessly inadequate invariant (see [2]).

If $X$ and $A$ are manifolds, then $X-A$ is an open manifold. For the constructions we have in mind, it would be more convenient if $X-A$ had a boundary. This may be achieved in a sense by removing from $X$ not only $A$ but a nice open neighborhood of $A$. The following paragraphs discuss this procedure in detail.

An embedding, $h$, of an orientable closed $k$-manifold $M^{k}$ in an orientable $n$-manifold $M^{n}$ will be called smooth if $h$ can be extended to an embedding of $M^{k} \times E^{n-k}$ in $M^{n}$, where $E^{n-k}$ is the closed unit ball in $n-k$ dimensional Euclidean space, and $M^{k}$ is identified with $M^{k}$ Xorigin. The embedding $h$ will be called semi-linear if both $M^{k}$ and $M^{n}$ are combinatorial manifolds, and $h$ is a simplicial homeomorphism of some rectilinear subdivision of $M^{k}$ onto a subcomplex of some rectilinear subdivision of $M^{n}$. If $M^{k}$ is already a subset of $M^{n}$, then $M^{k}$ will be said to be smoothly (semi-linearly) embedded in $M^{n}$ if the inclusion mapping is smooth (semi-linear).

Suppose $M^{k}$ has a neighborhood $M^{k} \times E^{n-k}$ in $M^{n}$. Let $B^{n-k}$ be the open ball in $n-k$ space of radius $1 / 2$. Then $M^{n}-\left(M^{k} \times B^{n-k}\right)$ is called an exterior of $M^{k}$ in $M^{n}$. Thus if $M^{k}$ is smoothly embedded in $M^{n}$, then $M^{k}$ has an exterior in $M^{n}$ which is an orientable $n$-manifold with boundary. It is clear that when the boundary $M^{k} \times S^{n-k-1}$ is removed from an exterior of $M^{k}$, what remains is homeomorphic to $M^{n}-M^{k}$. When $n$ is larger than three, it is not generally known whether two different exteriors of $M^{k}$ in $M^{n}$ are homeomorphic. For much of what we shall do, it is not necessary to know that the exterior of $M^{k}$ in $M^{n}$ is uniquely defined. Whenever it becomes desirable to have a fixed exterior, we shall restrict ourselves to smooth, semi-linear embeddings of $M^{k}$ in $M^{n}$ for which there exists a homeomorphism of $M^{k} \times E^{n-k}$ onto a closed regular neighborhood of $M^{k}$ in the interior of $M^{n}$, taking $M^{k}$ Xorigin onto $M^{k}$. In such a case, we shall use the complement of the open

(2) The last occurrence of the word nice in this sentence is essential, for otherwise we could derive from the pair $(X, A)$ the mapping cylinder of the inclusion $A \subset X$. If $X$ and $A$ are manifolds, such a derived space would in general be a complete invariant for the knotting problem of $A$ in $X$. 
regular neighborhood of $M^{k}$ as the exterior of $M^{k}$ in $M^{n}$. It is necessary to note that in this situation the exterior of $M^{k}$ is independent of the regular neighborhood of $M^{k}$ chosen, for any two regular neighborhoods of $M^{k}$ in the interior of $M^{n}$ are connected by a semi-linear homeomorphism of $M^{n}$ onto itself taking the one neighborhood onto the other (see [4]).

2. The isotopy extension theorem. Suppose now that $A$ is smoothly embedded in $X$ and $A^{\prime}$ is smoothly embedded in $X^{\prime}$, in such a way that some exterior of $A$ in $X$ is homeomorphic to some exterior of $A^{\prime}$ in $X^{\prime}$ under a homeomorphism $h$. We ask when $h$ can be extended to a homeomorphism of $X$ onto $X^{\prime}$ taking $A$ onto $A^{\prime}$. To this end, we desire something like a homotopy extension theorem, which will tell us that the extendability of $h$ depends only on some class of mappings containing $h$.

The relevant concept is that of isotopy. Two homeomorphisms, $h$ and $h^{\prime}$, of $X$ onto $Y$ are called isotopic if there is a map

$$
H: X \times[0,1] \rightarrow Y
$$

such that $H(x, 0)=h(x), H(x, 1)=h^{\prime}(x)$, and $h_{t}$, defined by $h_{t}(x)=H(x, t)$, is a homeomorphism of $X$ onto $Y$ for all $t \in[0,1]$. The definition is sometimes relaxed by not requiring that $h_{t}$ be onto. Since $X$ will always be a closed manifold here, the two versions are equivalent.

Corresponding to the classical homotopy extension theorem of Borsuk, there is an isotopy extension theorem. The proof is straightforward and will not be given. The real difficulty comes in applying the theorem, for it is usually much harder to show that two homeomorphisms are isotopic than it is to show that they are homotopic.

TheOREM (2.1). Isotopy EXTENSION THEOREM. Let $A \subset X$ have a neighborhood of the form $A \times[0,1]$ in which $A$ appears as $A \times 0$. Let $A \times[0,1)$ be open in $X$ and $A \times[0,1]$ its closure. Let $h: A \rightarrow A$ be a homeomorphism which extends to a homeomorphism $g:(X, A) \rightarrow(X, A)$. Let $h^{\prime}$ be a homeomorphism of $A$ which is isotopic to $h$ under the isotopy $H$. Then $h^{\prime}$ extends to a homeomorphism $g^{\prime}$ of $(X, A)$ which is isotopic to $g$ under an isotopy $G$ which extends $H$.

3. The annulus theorem. Of importance in classifying the homeomorphisms of a manifold with respect to isotopy is the annulus theorem, to be stated below. It is intimately connected with the isotopy classification of the homeomorphisms of the $n$-sphere. A proof of this theorem in all dimensions has not been found. Both the annulus theorem and the isotopy classification theorem for the homeomorphisms of $S^{n}$ can be proved using the additional hypothesis that certain submanifolds of $S^{n}$ can be triangulated. Since threedimensional manifolds can be triangulated, the results obtained will be sufficient for the particular problem discussed in chapter two.

Let $S^{n-1}$ be a smooth $n-1$ sphere in the $n$-sphere $S^{n}$. Then the closures of the complementary domains of $S^{n-1}$ are closed $n$-cells [5]. Suppose now that $S_{1}^{n-1}$ and $S_{2}^{n-1}$ are disjoint smooth $n-1$ spheres in $S^{n}$. Then the union of $S_{1}^{n-1}$ and $S_{2}^{n-1}$ has three complementary domains in $S^{n}$ (four, if $n=1$, but this 
case needs no further explanation). Let $A$ denote the closure of that domain which has both $n-1$ spheres for its boundary. $A$ is an $n$-manifold with boundary, which we shall call an annular region. It is conjectured that any annular region is homeomorphic to $S^{n-1} \times[0,1]$. The basis for this conjecture lies in the fact that it can be proved under the additional assumption that the $n-1$ spheres are semi-linearly embedded in the $n$-sphere.

The isotopy classification theorem for the $n$-sphere states that there are just two isotopy classes of homeomorphisms of the $n$-sphere, those of degree 1 and those of degree -1 .

THEOREM (3.1). Suppose that, for $k \leqq n$, every annular region in the $k$-sphere can be triangulated. Then $\left(1_{k}\right)$ and $\left(2_{k}\right)$ below are true for $k \leqq n$.

$\left(1_{k}\right)$ Every annular region in the $k$-sphere is homeomorphic to $S^{k-1} \times[0,1]$.

$\left(2_{k}\right)$ Every homeomorphism of $S^{k}$ which is homotopic to the identity (i.e., of degree +1$)$ is also isotopic to the identity.

In this paper we will be most interested in $\left(1_{3}\right)$, which follows directly from $[8 ; 30]$. For this reason, the somewhat long proof of Theorem (3.1), which may be found in the author's thesis, is omitted here. Also note that the hypothesis for Theorem (3.1) with $n=3$ is established by $[7 ; 8]$. Thus $\left(1_{k}\right)$ and $\left(2_{k}\right)$ hold for $k \leqq 3$. $\left(2_{3}\right)$ is included in the doctoral thesis of D. E. Sanderson and also follows from a paper of $G$. Fisher [10].

4. The general strategy. Let $M^{k}$ be a closed orientable $k$-manifold smoothly embedded in the orientable $n$-manifold $M^{n}$. Consider an exterior $M^{n}-\left(M^{k} \times B^{n-k}\right)$ of $M^{k}$ in $M^{n}$. Let $M_{1}^{k}$ be a copy of $M^{k}$, smoothly embedded in $M_{1}^{n}$, a copy of $M^{n}$. Let $M_{1}^{n}-\left(M_{1}^{k} \times B^{n-k}\right)$ be an exterior of $M_{1}^{k}$ in $M_{1}^{n}$. Suppose these two exteriors are homeomorphic. How close does this come to the homeomorphism of the pairs $\left(M^{n}, M^{k}\right)$ and $\left(M_{1}^{n}, M_{1}^{k}\right)$ ? Let $h$ be a homeomorphism from the former exterior to the latter. The extendability of $h$ to a homeomorphism of $\left(M^{n}, M^{k}\right)$ onto $\left(M_{1}^{n}, M_{1}^{k}\right)$ depends only on the restriction of $h$ to the boundary $M^{k} \times S^{n-k-1}$ of $M^{n}-\left(M^{k} \times B^{n-k}\right)$. We thus have a homeomorphism from the boundary of $M^{k} \times B^{n-k}$ to the boundary of $M_{1}^{k} \times B^{n-k}$ which we wish to extend over $M^{k} \times B^{n-k}$ so as to carry $M^{k} \times$ origin onto $M_{1}^{k} \times$ origin. We have thus temporarily removed $M^{n}$ from the problem. Sometimes $h$ can be extended in the desired way, sometimes it cannot. The extendability of $h$ depends only on the isotopy class of $h$, by Theorem (2.1). Thus instead of considering the group of all homeomorphisms of $M^{k} \times S^{n-k-1}$, we can factor out the normal subgroup consisting of those homeomorphisms which are isotopic to the identity. The quotient group, $\mathcal{H C}$, is the group of homeomorphism types of $M^{k} \times S^{n-k-1}$. It has also been called the homeotopy group of $M^{k} \times S^{n-k-1}$ (see [11]). Every smooth embedding of $M^{k}$ in $M^{n}$ with exterior homeomorphic to $M^{n}-\left(M^{k} \times B^{n-k}\right)$ can be obtained by identifying the boundary of $M^{k} \times B^{n-k}$ with the boundary of $M^{n}-\left(M^{k} \times B^{n-k}\right)$ via a homeomorphism $h$ in the group $G$ of all homeomorphisms of $M^{k} \times S^{n-k-1}$. The homeomorphism class of the resulting pair depends only on the isotopy class of $h$. Some identifications 
may not even give $M^{n}$ for the adjunction space, while other non-isotopic homeomorphisms may nevertheless give homeomorphic pairs. In any case, a natural beginning for the problem of deciding how many nonequivalent embeddings possess homeomorphic exteriors is the computation of the group TC of homeomorphism types of $M^{k} \times S^{n-k-1}$.

\section{THE EMBEDDING OF TWO-SPHERES IN THE FOUR-SPHERE}

5. Introductory remarks. The remainder of the paper will be devoted to the knotting problem for the $n$-sphere in the $n+2$ sphere. The case $n=2$ enjoys a crucial position in this family, and is the subject of the present chapter. The dimension is low enough to avoid most of the unpleasantries of higher dimensional Euclidean topology, yet just high enough to escape the difficult algebraic problems that hamper the investigation of ordinary knots in the three-sphere.

Let $S^{2}$ be a smoothly embedded two-sphere in the four-sphere, $S^{4} . S^{2}$ has a neighborhood of the form $S^{2} \times E^{2}$ with boundary $S^{2} \times S^{1}$. As explained in $\S 4$, we are interested in the group $\mathfrak{F}$ of homeomorphism types of $S^{2} \times S^{1}$. Recall that an element of $\mathfrak{H C}$ is an isotopy class of homeomorphisms of $S^{2} \times S^{1}$. A homeomorphism of $S^{2} \times S^{1}$ induces an automorphism of $H_{1}\left(S^{2} \times S^{1} ; Z\right) \approx Z$ and an automorphism of $H_{2}\left(S^{2} \times S^{1} ; Z\right) \approx Z$, each of which depends only on the isotopy class of the homeomorphism. The automorphism group in each case is $Z_{2}$, so we get a homomorphism $\varphi$ : $\mathfrak{H C} \rightarrow Z_{2}+Z_{2}$.

Let $r: S^{2} \rightarrow S^{2}$ be the antipodal map, and $s: S^{1} \rightarrow S^{1}$ the map induced on the unit circle in the complex plane by complex conjugation. Let $3 \mathcal{C}^{\prime} \approx Z_{2}+Z_{2}$ be the subgroup of $\mathfrak{H}$ generated by the isotopy classes of the map $(1,1)$, $(1, s),(r, 1)$, and $(r, s)$. Let $\rho$ be the isomorphism of $Z_{2}+Z_{2}$ with the subgroup $\mathcal{F C}^{\prime}$ of $\mathfrak{F C}$ determined by the condition $\varphi \rho=1$.

The main result of this chapter will be that the kernel of $\varphi$ is isomorphic to $Z_{2}$. But a normal subgroup of order two is central. This and the fact that $\varphi$ splits implies that $\mathfrak{H C} \approx Z_{2}+Z_{2}+Z_{2}$.

Theorem (5.1). $\mathfrak{H}\left(S^{2} \times S^{1}\right) \approx Z_{2}+Z_{2}+Z_{2}$.

As remarked above, it remains to be shown that $\operatorname{ker} \varphi \approx Z_{2}$.

6. Outline of the proof. For the purposes of the proof, we shall think of $S^{2}$ as the unit sphere in three-space, and of $S^{1}$ as the space of real numbers modulo 1 . Denote by 1 the identity homeomorphism of $S^{2} \times S^{1}$. Let $\Phi_{\alpha}$ denote a rotation of $S^{2}$ about a diameter through the north and south poles through an angle $2 \pi \alpha$ in some fixed direction. Define the homeomorphism $T$ of $S^{2} \times S^{1}$ by

$$
T(x, t)=\left(\Phi_{t}(x), t\right) .
$$

By the type of a homeomorphism of $S^{2} \times S^{1}$ we shall mean the image of its isotopy class under $\varphi$. Thus the kernel of $\varphi$ consists of the isotopy classes of the homeomorphisms of type $(1,1)$. Both 1 and $T$ are of type $(1,1)$. We will 
show that any homeomorphism of $S^{2} \times S^{1}$ of type $(1,1)$ is isotopic either to 1 or to $T$. Let $h$ be any homeomorphism of $S^{2} \times S^{1}$ of type $(1,1)$. In stage one of the proof, we will deform $h$ isotopically until $h / S^{2} \times 0$ is the identity. In stage two, we further deform $h$ isotopically until $h / S^{2} \bigvee S^{1}$ is the identity, where $S^{2} \bigvee S^{1}=\left(S^{2} \times 0\right) \cup\left(\right.$ north pole $\left.\times S^{1}\right)$. Now let $C$ be a small circle on $S^{2}$ about the north pole. In stage three we deform $h$ isotopically until $h / S^{2} \times 0$ is the identity and $h$ takes $C \times S^{1}$ onto itself. In the fourth stage we further deform $h$ until in addition to having the properties attained in stage three, $h$ also has the form of a "standard" $n$-tuple twist on $C \times S$. In the fifth stage, we deform $h$ until $h / S^{2} \times 0$ is the identity and $h$ takes $C \times S^{1}$ onto itself either by the identity or by a single twist in a fixed direction. In stage six, we deform $h$ either into 1 or into $T$. The final stage is devoted to showing that $T$ is not homotopic to 1 ; a fortiori, not isotopic to 1 .

7. A preliminary theorem. Denote the closed interval $[0,1]$ by $I$. In this section we give the isotopy classification of the homeomorphisms of $S^{1} \times I$ whose restrictions to the boundary $B=\left(S^{1} \times 0\right) \cup\left(S^{1} \times 1\right)$ are the identity. Note that a point of $S^{1} \times I$ is a pair of real numbers, the first number being taken $\bmod 1$ and the second restricted to the interval $[0,1] . G^{b}$ will denote the group of homeomorphisms of $S^{1} \times I$ whose restrictions to $B$ are the identity. $G^{b}$ is topologized by the compact-open topology. $G_{0}^{b}$ will denote the arcwise connected component of the identity in $G^{b}$. Every homeomorphism in $G^{b}$ is isotopic to the identity, but the isotopies will in general move points on $B$. An isotopy which moves no points on $B$ is called a $B$-isotopy. $G_{0}^{b}$ is then the set of homeomorphisms of $S^{1} \times I$ which are $B$-isotopic to the identity.

Let $h \in G^{b}$. We are going to define the winding number, $w(h)$. Let $\eta$ be the isomorphism of $\pi_{1}\left(S^{1}, 0\right)$ with $Z$ which takes the class of the path $f(t)=t$ onto 1 . Let $\alpha$ be any path in $S^{1} \times I$ from $(0,0)$ to $(0,1)$. Let $p_{1}: S^{1} \times I \rightarrow S^{1}$ be the natural projection. Then $p_{1} \alpha$ is a closed path in $S^{1}$ based at 0 . Hence $\left[p_{1} \alpha\right]$ is an element of $\pi_{1}\left(S^{1}, 0\right)$ and $\eta\left[p_{1} \alpha\right]=w(\alpha)$ is an integer, called the winding number of the path $\alpha$. Now take $h \in G^{b}$ and consider the number $w(h \alpha)-w(\alpha)$.

\section{Lemma (7.1). $w(h \alpha)-w(\alpha)$ is independent of $\alpha$.}

First note that if $\alpha$ is homotopic to $\alpha^{\prime}$ (relative to end points), then $w(\alpha)$ $=w\left(\alpha^{\prime}\right)$. Let $\zeta$ be the path defined by $\zeta(t)=(0, t)$. Then $\alpha$ is homotopic to $\zeta \beta$, where $\beta$ is a closed path in $S^{1} \times 1$ based at $(0,1)$. Now $h \alpha$ is homotopic to $h(\zeta \beta)=h(\zeta) h(\beta)=h(\zeta) \beta$. Since $w(\alpha)=w(\zeta \beta)=w(\zeta)+\eta\left[p_{1} \beta\right]$ and $w(h \alpha)$ $=w(h(\zeta) \beta)=w(h \zeta)+\eta\left[p_{1} \beta\right], w(h \alpha)-w(\alpha)=w(h \zeta)-w(\zeta)$, where $\zeta$ is a fixed path independent of $\alpha$. This proves the lemma.

We now define the winding number of $h, w(h)$, to be $w(h \alpha)-w(\alpha)$ for any path $\alpha$ in $S^{1} \times I$ from $(0,0)$ to $(0,1)$. Then $w\left(h \cdot h^{\prime}\right)=w\left(h \cdot h^{\prime} \alpha\right)-w(\alpha)=w\left(h \cdot h^{\prime} \alpha\right)$ $-w\left(h^{\prime} \alpha\right)+w\left(h^{\prime} \alpha\right)-w(\alpha)=w(h)+w\left(h^{\prime}\right)$. Thus $w$ defines a homomorphism $w: G^{b} \rightarrow Z$. 
ThEOREM (7.2). The kernel of $w$ is $G_{0}^{b}$.

$B$-isotopic homeomorphisms in $G^{b}$ have the same winding number. Hence $G_{0}^{b}$ is contained in the kernel of $w$. We must show that an arbitrary homeomorphism in $G^{b}$ with winding number zero is $B$-isotopic to the identity. The crux of the proof is to deform $h \in \operatorname{ker} w B$-isotopically until $h / \zeta$ is the identity. Cutting $S^{1} \times I$ along $\zeta$ yields a two-cell, on which $h$ acts in the obvious way. But $h$ is the identity on the boundary of this two-cell, and hence is isotopic to the identity under an isotopy which is stationary on the boundary (see [6]). This isotopy may be interpreted as a $B$-isotopy of $h$ on $S^{1} \times I$.

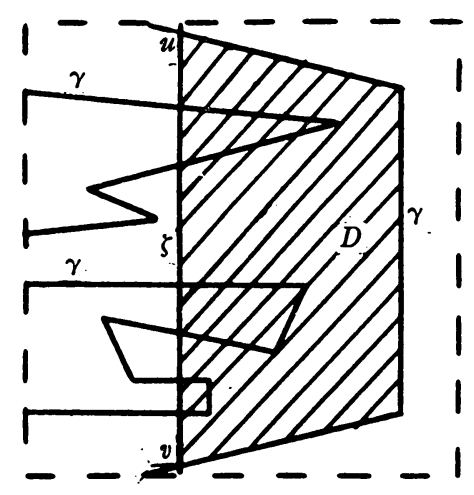

$1 \mathrm{a}$

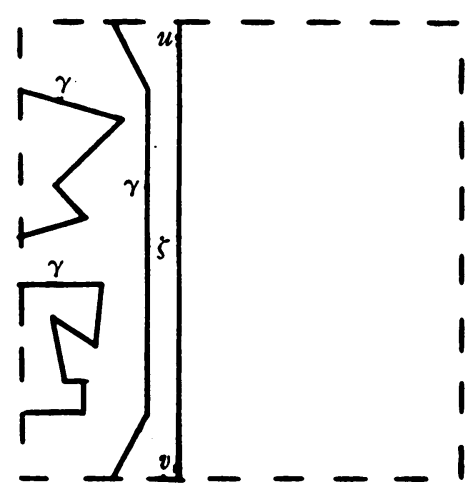

$1 \mathrm{~b}$

FIGURE 1

The procedure of deforming $h$ until $h / \zeta$ is the identity is nothing more than an exercise in the use of the Schönflies theorem (see, for example, [12]). We content ourselves here with an outline of this process. Let $\gamma$ be a polygonal arc in $S^{1} \times I$ which meets $B$ only in $(0,0)$ and $(0,1)$, and which is close enough to $h \zeta$ so that $h^{-1} \gamma$ does not meet $(1 / 2) \times I$. Then, using a technique described in detail in [13], $h$ may be deformed $B$-isotopically so that $h$ carries $\zeta$ onto $\gamma$. Since $w(\zeta)=0, w(\gamma)=0 . \gamma$ is then slightly deformed, $B$-isotopically, so that all of its intersections with $\zeta$ are actual crossings, rather than tangencies or coincidences. Proceeding along $\gamma$ from $(0,0)$ to $(0,1)$, we pass through various intersections with $\zeta$, some of these from the "left," others from the "right" (not forgetting the intersections at $(0,0)$ and $(0,1)$ ). If there are any interior intersections, then there must be a pair of intersections, $u$ and $v$, one from the right and the other from the left, which are adjacent on $\gamma$, and at least one of which is not on the boundary $B$. The situation is shown in Figure 1a. The simple closed curve formed by the arcs of $\zeta$ and $\boldsymbol{\gamma}$ between $u$ and $v$ bounds a two-cell $D$ in $S^{1} \times I . S^{1} \times I$ is deformed $B$-isotopically so that the arc of $\gamma$ between $u$ and $v$ slides over $D$ to the other side of $\zeta$, thus removing at least one of the intersections $u$ and $v$ (and both if $u$ and $v$ are interior intersections). During the deformation, all intersections between 
$u$ and $v$ on $\zeta$ are also removed (see Figure $1 \mathrm{~b}$ ). This procedure is iterated until $\gamma$ meets $\zeta$ only at $(0,0)$ and $(0,1)$. The technique of $[13]$ is then employed to deform $\gamma$ until it coincides with $\zeta$. Thus $h$ has been deformed $B$-isotopically until $h(\zeta)=\zeta$. But any homeomorphism of an arc which leaves the end points fixed is isotopic to the identity (under the obvious linear isotopy). This isotopy extends to a $B$-isotopy of $S^{1} \times I$, and so finally $h / \zeta$ is the identity. This completes the proof. Theorem (7.2) states in effect that two homeomorphisms in $G^{b}$ are $B$-isotopic if and only if they have the same winding number.

8. Stage one. Let $h$ be a homeomorphism of $S^{2} \times S^{1}$ of type $(1,1)$. In this section we will deform $h$ isotopically until $h / S^{2} \times 0$ is the identity.

A subset $A^{\prime}$ of the metric space $X$ is said to be an $\epsilon$-approximation to the subset $A$ of $X$ if there exists a homeomorphism $f: A \rightarrow A^{\prime}$ which moves each point of $A$ a distance less than $\epsilon$.

Introduce a metric in $S^{2} \times S^{1}$.

LeMma (8.1). There is a number $\epsilon>0$ such that if $S$ is a smooth two-sphere in $S^{2} \times S^{1}$ which is an $\epsilon$-approximation to $S^{2} \times 0$, then there is a homeomorphism $f: S^{2} \times S^{1} \rightarrow S^{2} \times S^{1}$ which takes $S^{2} \times 0$ onto $S$ and which is isotopic to the identity.

The homeomorphism $f$ will be constructed as $f=f_{2} \cdot f_{1}$, where $f_{1}$ takes $S^{2} \times 0$ onto $S^{2} \times 1 / 2$ and $f_{2}$ takes $S^{2} \times 1 / 2$ onto $S$. Define $f_{1}$ by $f_{1}(x, t)=(x, t+1 / 2) . f_{1}$ is isotopic to the identity. Suppose now that we can construct a homeomorphism $g$ of $S^{2} \times S^{1}$ onto itself which takes $S^{2} \times 0$ onto $S$ and $S^{2} \times 1 / 2$ onto itself, but which we do not know is isotopic to the identity. Then $f_{2}=g f_{1} g^{-1}$ takes $S^{2} \times 1 / 2$ onto $S$ and is isotopic to the identity because $f_{1}$ is. Hence $f=f_{2} \cdot f_{1}$ takes $S^{2} \times 0$ onto $S$ and is isotopic to the identity, thus satisfying the conditions of the lemma.

It remains, therefore, to construct $g$. Cut $S^{2} \times S^{1}$ along $S^{2} \times 1 / 2$ and embed in three-space as the region between concentric spheres about the origin of radii 1 and 3 , with $S^{2} \times 0$ going into the sphere of radius 2. Choose $\epsilon$ so small that if $S$ is a smooth two-sphere in $S^{2} \times S^{1}$ and $k$ a homeomorphism of $S$ onto $S^{2} \times 0$ which moves no point more than $\epsilon$, then $S$ is disjoint from $S^{2} \times 1 / 2$ and the straight line segment between a point on $S$ and its image under $k$ (all viewed in three-space) lies in the region between the spheres of radii 1 and 3. Thus $S$ must separate the two copies of $S^{2} \times 1 / 2$, just as $S^{2} \times 0$ does, for $S$ is homotopic to $S^{2} \times 0$ in the annular region of three-space. The two regions of $S^{2} \times S^{1}$ bounded by $S$ and $S^{2} \times 1 / 2$ are each homeomorphic to $S^{2} \times I$ by $\left(1_{3}\right)$ of Theorem (3.1). Since the two regions of $S^{2} \times S^{1}$ bounded by $S^{2} \times 0$ and $S^{2} \times 1 / 2$ are also homeomorphic to $S^{2} \times I$, the construction of the homeomorphism $g$ is immediate, and the lemma is proved.

LemMa (8.2). Let $h$ be a homeomorphism of $S^{2} \times S^{1}$ onto itself. Then $h$ can be deformed isotopically until $h\left(S^{2} \times 0\right)$ is disjoint from $S^{2} \times 1 / 2$.

Triangulate $S^{2} \times S^{1}$ so that $S^{2} \times 0$ and $S^{2} \times 1 / 2$ appear as subcomplexes. 
By [14] there is a polyhedral two-sphere $S$ in $S^{2} \times S^{1}$ which is an $\epsilon$-approximation to $h\left(S^{2} \times 0\right)$, where $\epsilon$ is chosen so small that $h\left(S^{2} \times 0\right)$ can be deformed isotopically onto $S$. Deform $S$ slightly so that it is in general position with respect to $S^{2} \times 1 / 2$. We have thus far succeeded in deforming $h$ so that $h\left(S^{2} \times 0\right)$ is a polyhedral two-sphere whose intersection with $S^{2} \times 1 / 2$ consists of a number of disjoint simple closed curves. The technique of removing the intersections is a straightforward generalization of the technique employed in the proof of Theorem (7.2), as follows.

Let $C$ be an innermost curve of intersection on $S$, and let $D_{1}$ be a two-cell on $S$ bounded by $C$ which contains no other curves of intersection. $C$ divides $S^{2} \times 1 / 2$ into two two-cells. Let $D_{2}$ be that one which when added to $D_{1}$ forms a two-sphere which bounds a three-cell $E$ in $S^{2} \times S^{1}$. That $D_{2}$ can be so chosen is seen immediately by looking at the universal covering space of $S^{2} \times S^{1}$. $S$ is then deformed isotopically by sliding $D_{1}$ over $E$ to the other side of $S^{2} \times 1 / 2$. During this deformation, all the curves of intersection lying on $D_{2}$ are also removed. See Figures $2 \mathrm{a}$ and $2 \mathrm{~b}$. Iterating this procedure proves the lemma.

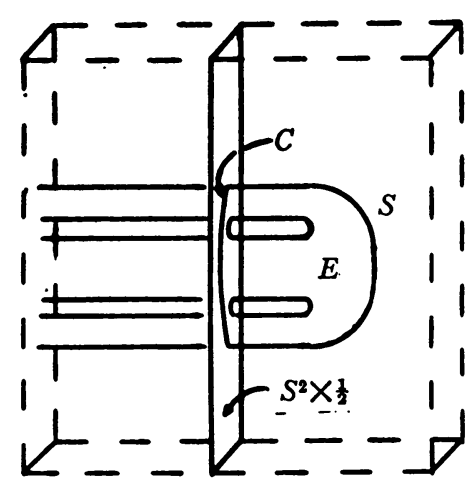

$2 a$

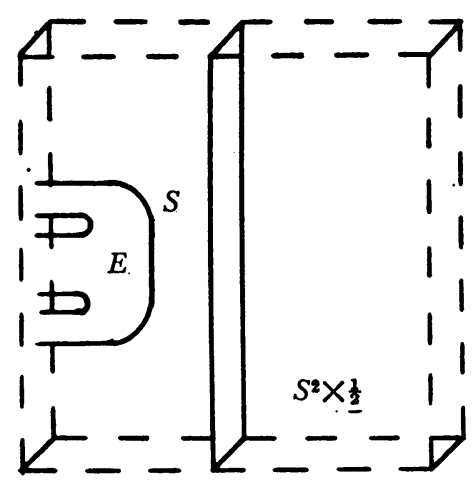

$2 \mathrm{~b}$

Figure 2

THEOREM (8.3). Let $h$ be a homeomorphism of $S^{2} \times S^{1}$ of type $(1,1)$. Then $h$ can be deformed isotopically until $h / S^{2} \times 0$ is the identity.

Using Lemma (8.2), $h$ can first be deformed isotopically until $h\left(S^{2} \times 0\right)$ is disjoint from $S^{2} \times 1 / 2$. Then the two regions of $S^{2} \times S^{1}$ bounded by $h\left(S^{2} \times 0\right)$ and $S^{2} \times 1 / 2$ are homeomorphic to $S^{2} \times I$ by $\left(1_{3}\right)$ of Theorem (3.1). As in Lemma (8.1), we may deform $h\left(S^{2} \times 0\right)$ isotopically onto $S^{2} \times 0$ by first deforming it onto $S^{2} \times 1 / 2$. Since $h$ is of type $(1,1), h$ now takes $S^{2} \times 0$ onto itself with degree +1 , and is thus isotopic to the identity by $\left(2_{2}\right)$ of Theorem (3.1). Extending this isotopy to $S^{2} \times S^{1}$ by taking the product with the identity map on $S^{1}$ finally deforms $h$ so that $h / S^{2} \times 0$ is the identity. Note that the theorem also holds if $h$ is of type $(1,-1)$. 
9. Stage two. In this section we begin with a homeomorphism $h$ of $S^{2} \times S^{1}$ of type $(1,1)$, whose restriction to $S^{2} \times 0$ is the identity. We will deform $h$ isotopically until $h / S^{2} \bigvee S^{1}$ is the identity.

Since $h / S^{2} \times 0$ is the identity, we may cut $S^{2} \times S^{1}$ along $S^{2} \times 0$ and interpret $h$ as a homeomorphism of $S^{2} \times I$ whose restriction to the boundary $B$ of $S^{2} \times I$ is the identity (for this it is essential that $h$ be of type $(1,1)$ rather than of type $(1,-1))$. Let $x_{n}$ and $x_{s}$ denote the north and south poles of $S^{2}$. We will proceed to deform $h B$-isotopically (see $\$ 7$ ) until $h / x_{n} \times I$ is the identity. Such a $B$-isotopy can be interpreted as an isotopy on $S^{2} \times S^{1}$ which is stationary on $S^{2} \times 0$, and the desired result will have been obtained. In order to interpret an isotopy of $h$ on $S^{2} \times I$ as an isotopy on $S^{2} \times S^{1}$, it is only necessary that this isotopy behave identically on the two boundary spheres of $S^{2} \times I$. Nevertheless, we restrict ourselves to $B$-isotopies in order to preserve the analogy with the results of $\S 7$.

Suppose for the moment that the following lemma has been proved.

Lemma (9.1). Let $\alpha$ be a polygonal arc in $S^{2} \times I$ from $\left(x_{n}, 0\right)$ to $\left(x_{n}, 1\right)$ which meets $B$ only at its endpoints. Then there is a $B$-isotopy of $S^{2} \times I$ taking $\alpha$ onto $x_{n} \times I$.

Let $x^{\prime}$ be a point of $S^{2}$ close to the north pole $x_{n}$. Since $h\left(x_{n} \times I\right)$ is of dimension 1 , it cannot disconnect $\left(x^{\prime}, 0\right)$ from $\left(x^{\prime}, 1\right)$. Hence let $\alpha$ be a polygonal arc from $\left(x^{\prime}, 0\right)$ to $\left(x^{\prime}, 1\right)$ in $S^{2} \times I$ which meets $B$ only at its endpoints, and which is disjoint from $h\left(x_{n} \times I\right)$. We are not concerned with intersections of $\alpha$ and $x_{n} \times 1$, although they can easily be avoided.

The set $B \cup \alpha$ is locally tame (see [15]), and since $B \cup h\left(x_{n} \times I\right)$ is the image of $B \cup\left(x_{n} \times I\right)$ under a homeomorphism of $S^{2} \times I, B \cup h\left(x_{n} \times I\right)$ is also locally tame. Since $\alpha$ is disjoint from $h\left(x_{n} \times I\right)$, the set $B \cup \alpha \cup h\left(x_{n} \times I\right)$ is locally tame. Thus by [15] there is a triangulation of $S^{2} \times I$ in which both $\alpha$ and $h\left(x_{n} \times I\right)$ appear as polygonal arcs, although $x_{n} \times I$ may not be polygonal in this triangulation.

Let $H_{1}$ be an isotopy (not $B$-isotopy) of $S^{2} \times I$ which takes $h\left(x_{n} \times I\right)$ onto a polygonal arc $\gamma$ (in the new triangulation) from $\left(x^{\prime}, 0\right)$ to $\left(x^{\prime}, 1\right) . H_{1}$ can at least be chosen to behave identically on the two boundary spheres. Two applications of Lemma (9.1) yield a $B$-isotopy $H_{2}$ taking $\gamma$ onto $\alpha$. But now returning to our original affine structure (induced by some fixed inclusion of $S^{2} \times I$ in three-space), we can find an isotopy (not $B$-isotopy) $H_{3}$ taking $\alpha$ onto a polygonal arc $\beta$ from $\left(x_{n}, 0\right)$ to $\left(x_{n}, 1\right) . H_{3}$ can also be chosen to behave identically on the two boundary spheres. A final application of Lemma (9.1) deforms $\beta B$-isotopically onto $x_{n} \times I$, via the $B$-isotopy $H_{4}$. So far $h\left(x_{n} \times I\right)$ has been deformed back onto $x_{n} \times I$. A final $B$-isotopy $H_{5}$ deforms $h$ so that $h / x_{n} \times I$ is the identity. The compound isotopy $H=H_{1} H_{2} H_{3} H_{4} H_{5}$ is not a $B$-isotopy, but does behave identically on the two boundary spheres of $S^{2} \times I$. Let $G$ be an isotopy which behaves on every $S^{2} \times t$ as $H$ behaves on the bound- 
ary spheres. Then $G$ begins and ends at the identity. Hence $G^{-1} \cdot H$ is a $B$ isotopy which accomplishes just what $H$ accomplished, namely to deform $h$ so that $h / x_{n} \times I$ is the identity.

We turn now to the proof of the lemma. For convenience we think of $S^{2} \times I$ as the closed region between the spheres of radii 1 and 2 about the origin in three-space. We first deform $\alpha B$-isotopically so that when we look at $S^{2} \times I$ from one side, no part of $\alpha$ is hidden behind $S^{2} \times 0$ (the inner sphere in three-space). Slightly displacing the vertices of $\alpha$ leaves only double "crossings" (see Figure 3a). Proceeding inward along $\alpha$ from $\left(x_{n}, 1\right)$, we come to the first crossing (see Figure $3 \mathrm{~b}$ ). We deform the dotted arc $(a b)$ into the solid arc $(a b)$ by slipping it half way around a neighborhood of $S^{2} \times 1$. This reduces the number of crossings by one, and when repeated finally deforms $\alpha B$ isotopically so that $\alpha$ has no crossing points when viewed from one side. Then

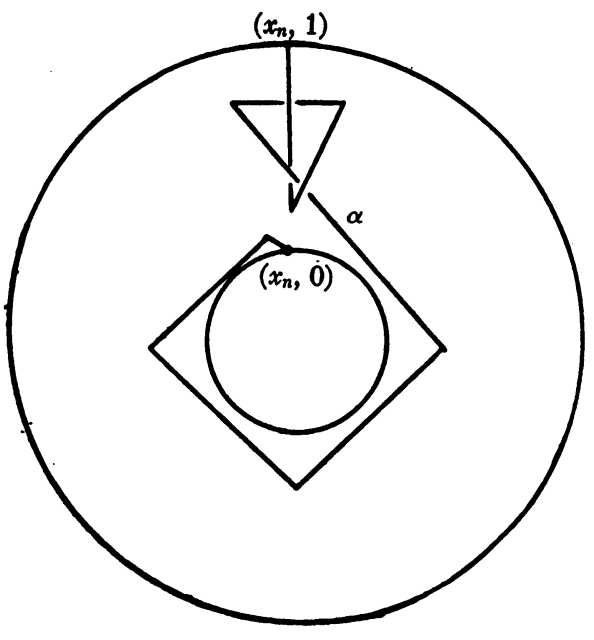

$3 a$

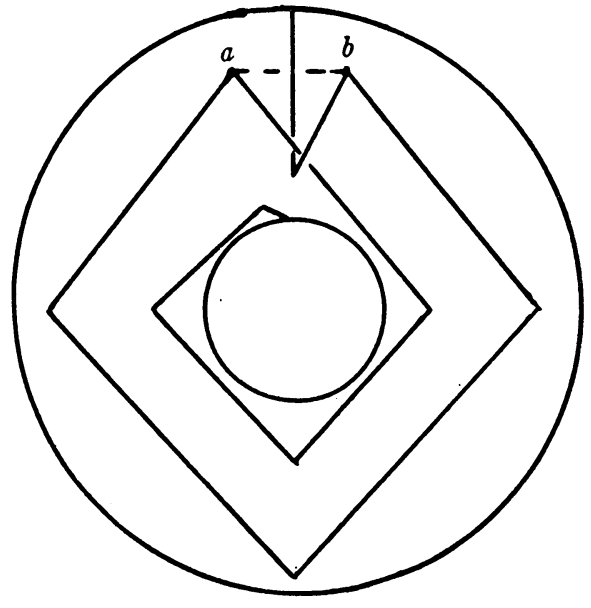

$3 \mathrm{~b}$

FIGURE 3

$\alpha$ is deformed $B$-isotopically onto the plane of the paper, during which deformation the points on $\alpha$ are either stationary or move horizontally. Using Theorem (7.2), $\alpha$ is deformed $B$-isotopically into the standard form of a spiral arc circling about the intersection of $S^{2} \times I$ and the plane of the paper as it proceeds from $\left(x_{n}, 0\right)$ to $\left(x_{n}, 1\right)$. Lifting this arc several times over $S^{2} \times 0$ finally places $\alpha$ in coincidence with $x_{n} \times I$, thus proving Lemma (9.1).

The results of this section are expressed in the following theorem.

TheOREM (9.2). Let $h$ be a homeomorphism of $S^{2} \times S^{1}$ of type $(1,1)$ whose restriction to $S^{2} \times 0$ is the identity. Then $h$ can be deformed isotopically until $h / S^{2} \bigvee S^{1}$ is the identity.

10. Stage three. We begin now with a homeomorphism $h$ of $S^{2} \times S^{1}$ such 
that $h / S^{2} \bigvee S^{1}$ is the identity. Let $C$ be a small circle on $S^{2}$ about the north pole $x_{n}$, and $C^{\prime}$ a slightly larger concentric circle. Let $D$ and $D^{\prime}$ be the two-cells bounded by these circles on $S^{2}$ which contain $x_{n}$. As in $\$ 9$, interpret $h$ as a homeomorphism of $S^{2} \times I$. Keeping $C^{\prime}$ fixed, make $C$ so small that $h(D \times I)$ $C\left(\right.$ Int $\left.D^{\prime}\right) \times I$. Let $T=\left(D^{\prime} \times I\right)-h(($ Int $D) \times I)$.

LEMMA (10.1). $T$ is a solid torus.

The boundary of $T$ is locally tame, hence tame [15], and is easily seen to be a torus. Then by Dehn's Lemma [16], $T$ is a solid torus if and only if $\pi_{1}(T)=Z$.

But $C \times I$ is a deformation retract of $\left(D-x_{n}\right) \times I$, and $h / x_{n} \times I$ is the identity. Hence $h(C \times I)$ is a deformation retract of $h(D \times I)-x_{n} \times I$. Thus $T$ is a deformation retract of $\left(D^{\prime}-x_{n}\right) \times I$. But $\pi_{1}\left(\left(D^{\prime}-x_{n}\right) \times I\right)=Z$. Hence $\pi_{1}(T)=Z$, and the lemma is proved.

From the retraction, one sees that $h(C \times I)$ carries a generator of $\pi_{1}(T)$. Consequently there is a homeomorphism of $D^{\prime} \times I$ onto itself which carries $h(C \times I)$ onto $C \times I$ and is the identity on the boundary of $D^{\prime} \times I$. By [6], such a homeomorphism is isotopic to the identity under an isotopy which is stationary on the boundary of $D^{\prime} \times I$. Such an isotopy therefore extends, via the identity, to a $B$-isotopy of $S^{2} \times I$ which carries $h(C \times I)$ back onto $C \times I$. Interpreting these results on $S^{2} \times S^{1}$, we obtain the following theorem.
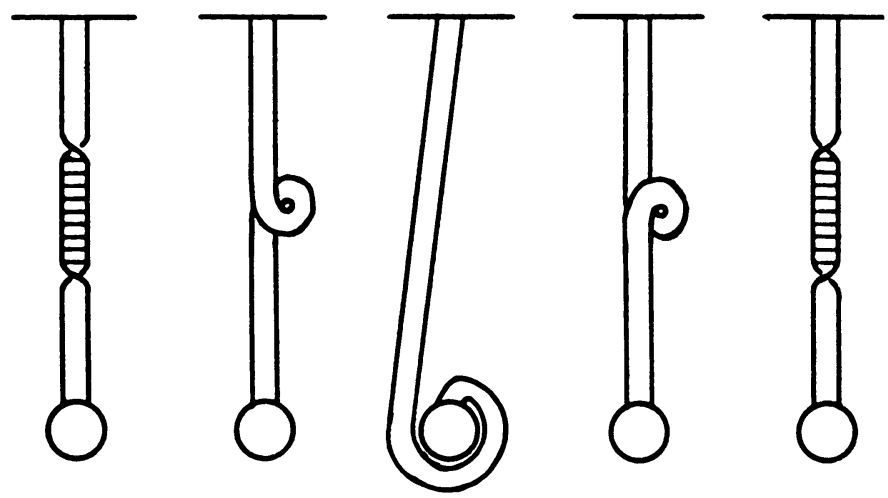

FIGURE 4

THEOREM (10.2). Let $h$ be a homeomorphism of $S^{2} \times S^{1}$ such that $h / S^{2} \bigvee S^{1}$ is the identity. Let $C$ be a small circle on $S^{2}$ about the north pole, $x_{n}$. Then $h$ can be deformed isotopically until $h / S^{2} \times 0$ is the identity and $h$ takes $C \times S^{1}$ onto itself.

11. Stage four. Looking at $S^{2} \times I$ again, assume that $h$ is the identity on $B$ and takes $C \times I$ onto itself. By Theorem (7.2), $h / C \times I$ is isotopic to a standard $n$-tuple twist. If for example we parametrize $C$ by the angle $\phi \bmod 2 \pi$, then $h / C \times I$ is isotopic to one of the maps $f_{n}(\phi, t)=(\phi+2 \pi n t, t)$. This $B$ - 
isotopy on $C \times I$ clearly extends to a $B$-isotopy on $S^{2} \times I$, and hence may be interpreted as an isotopy on $S^{2} \times S^{1}$. This completes stage four.

12. Stage five. In this stage we continue to deform $h$ until either $h / C \times I$ is the identity (i.e., $f_{0}$ ) or $h / C \times I=f_{1}$, according as the $n$ in stage four is even or odd. Figure 4 shows one part of this deformation process, in which $n$ is changed by two. This process, repeated sufficiently often, completes stage five.

13. Stage six. Let $K=\left(S^{2} \times 0\right) \cup\left(S^{2} \times 1\right) \cup(C \times I)$. Starting with the homeomorphism $h$ of $S^{2} \times S^{1}$ of type $(1,1)$, we first deformed $h$ isotopically until $h / S^{2} \times 0$ was the identity, thus permitting us to interpret $h$ as a homeomorphism of $S^{2} \times I$. The identity homeomorphism 1 and the homeomorphism $T$ of $S^{2} \times S^{1}$, described in $\S 6$, both restrict to the identity on $S^{2} \times 0$, and may thus be interpreted as homeomorphisms of $S^{2} \times I$. The results up to this point may be summarized by saying that if $h$ is a homeomorphism of $S^{2} \times I$ whose restriction to the boundary $B$ is the identity, then $h$ can be deformed $B$ isotopically until $h / K$ is either the identity or coincides with $T / K$.

Lemma (13.1). Let $f$ and $g$ be two homeomorphisms of $S^{2} \times I$ whose restrictions to $B$ are the identity and which agree on $C \times I$. Then $f$ and $g$ are $B$-isotopic.

For $f^{-1} g / K$ is the identity. But $S^{2} \times I-K$ consists of two disjoint open three-cells whose boundaries are nonsingular and contained in $K$. Since $f^{-1} g$ cannot interchange these three-cells, the restriction to each cell is a homeomorphism which is the identity on the boundary and hence isotopic to the identity under an isotopy which is stationary on the boundary by [6]. Putting these two isotopies together yields a $B$-isotopy of $f^{-1} g$ with the identity. Hence $f$ is $B$-isotopic to $g$.

Hence viewed on $S^{2} \times I$, either $h$ is $B$-isotopic to the identity or $h$ is $B$ isotopic to $T$. Reinterpreting our results on $S^{2} \times S^{1}$ yields the following theorem.

THEOREM (13.2). Let $h$ be a homeomorphism of $S^{2} \times S^{1}$ of type $(1,1)$. Then either $h$ is isotopic to the identity or else $h$ is isotopic to the homeomorphism $T$ described in $\$ 6$.

14. Stage seven. In this final stage we will show that $T$ is not homotopic to 1 , and, a fortiori, not isotopic to 1 . Denote by $p_{1}$ the projection of $S^{2} \times S^{1}$ onto $S^{2}$ and by $p_{2}$ the projection of $S^{2} \times S^{1}$ on $S^{1}$. Then $T$ is homotopic to 1 if and only if $p_{1} \cdot T$ is homotopic to $p_{1}$ and $p_{2} \cdot T$ is homotopic to $p_{2}$. But $p_{2} \cdot T=p_{2}$, hence we must show that $p_{1} \cdot T$ is not homotopic to $p_{1}$. To do this, we use the classification of maps of a three-dimensional complex into the twosphere given by Pontrjagin [17]. Let $y^{2}$ be a generator of $H^{2}\left(S^{2} ; Z\right)$. Then a necessary condition for $p_{1} \cdot T$ to be homotopic to $p_{1}$ is that $\left(p_{1} \cdot T\right) *\left(y^{2}\right)$ $=p_{1} *\left(y^{2}\right) \in H^{2}\left(S^{2} \times S^{1} ; Z\right)$. This condition is satisfied, for $\left(p_{1} \cdot T\right) *\left(y^{2}\right)$ $=p_{1} *\left(y^{2}\right)=z^{2}$ is a generator of $H^{2}\left(S^{2} \times S^{1} ; Z\right)$. Hence if $S^{2} \times S^{1}$ is triangulated as a simplicial complex, $p_{1} \cdot T$ can be deformed homotopically until it coin- 
cides with $p_{1}$ on the two-skeleton of $S^{2} \times S^{1}$. Thus we define a difference cochain $d^{3}\left(p_{1}, p_{1} \cdot T\right)$ which represents an element $z^{3}$ of $H^{3}\left(S^{2} \times S^{1} ; \pi_{3}\left(S^{2}\right)\right)$. Choose a fixed isomorphism of $\pi_{3}\left(S^{2}\right)$ with $Z$ so that we may consider $z^{3}$ as an element of $H^{3}\left(S^{2} \times S^{1} ; Z\right)$. The theorem of Pontrjagin then states that $p_{1} \cdot T$ is homotopic to $p_{1}$ if and only if there exists a one-dimensional cohomology class $z^{1} \in H^{1}\left(S^{2} \times S^{1} ; Z\right)$ such that

$$
z^{3}=2 z^{1} \cup z^{2}
$$

In order to demonstrate that $p_{1} \cdot T$ is not homotopic to $p_{1}$, we must show that $z^{3}$ is an odd multiple of a generator of $H^{3}\left(S^{2} \times S^{1} ; Z\right)$. For the purposes of computation it is most convenient to consider $S^{2} \times S^{1}$ as a $C W$-complex with a single cell in each dimension up through three. It is easily seen that we get the same answers this way as by using a simplicial complex. Decompose $S^{1}$ into a one-cell with collapsed boundary plus a zero-cell. Similarly decompose $S^{2}$ into a two-cell with collapsed boundary plus a zero-cell. Form $S^{2} \bigvee S^{1}$ by identifying the zero-cells. This gives us the two-skeleton of $S^{2} \times S^{1}$. Now take a closed three-cell and collapse an annular region on its boundary to an arc in the obvious way. Its boundary now consists of a pair of two-spheres joined by an arc. Identify the two-spheres so that the ends of the arc come together. The boundary of the three-cell is now an $S^{2} \bigvee S^{1}$, which is the twoskeleton of our $C W$-decomposition of $S^{2} \times S^{1}$. Thus the three-cell with the above identifications on its boundary gives us $S^{2} \times S^{1}$.

Let $S^{3}$ be a three-sphere with upper hemisphere $E_{+}^{3}$ and lower hemisphere $E_{-}^{3}$ and equatorial two-sphere $S^{2}$. Collapse the equatorial two-sphere to $S^{2} \bigvee S^{1}$ in the manner indicated in the above paragraph. We thus obtain two copies of $S^{2} \times S^{1}$ joined along $S^{2} \bigvee S^{1}$. Map one copy, say that determined by $E_{+}^{3}$, into $S^{2}$ by the map $p_{1}$. Map the other copy into $S^{2}$ by the map $p_{1} \cdot T$. Since $T$ is the identity on $S^{2} \bigvee S^{1}$, we obtain a well-defined map $f: S^{3} \rightarrow S^{2}$. The cohomology class $z^{3}$ is an odd multiple of a generator of $H^{3}\left(S^{2} \times S^{1} ; Z\right)$ if and only if the Hopf invariant of $f$ is odd.

Choose two points, $a$ and $b$, on $S^{2}$ other than $x_{n}$. Let $A$ and $B$ be the complete inverse images of $a$ and $b$ under $f . A$ and $B$ are simple closed curves, and the Hopf invariant of $f$ is given, up to sign, by their linking number. Let $A \cap E_{+}^{3}=A_{+}$, and so forth. Figure 5 a shows $A_{+}$and $B_{+}$in $E_{+}^{3} ;$ Figure $5 \mathrm{~b}$ shows $A_{-}$and $B_{-}$in $E_{-}^{3}$. In Figure $5 \mathrm{c}$ we have deformed $A_{-}$and $B_{-}$isotopically in $E_{-}^{3}$. Using Figures $5 \mathrm{a}$ and $5 \mathrm{c}$, the construction of a disc bounded by $B$ and intersected exactly once by $A$ is immediate. Hence the Hopf invariant of $f$ is \pm 1 , and thus $p_{1} \cdot T$ is not homotopic to $p_{1}$. This completes the final stage in the proof of Theorem (5.1).

We have, in addition, proved the following theorem.

Theorem (14.1). Two homeomorphisms of $S^{2} \times S^{1}$ are isotopic if and only if they are homotopic. 
15. Two-spheres in the four-sphere with a given exterior. Let $S^{2}$ be a smooth two-sphere in the four-sphere $S^{4}$. Let $E^{2}$ denote a closed two-cell and $B^{2}$ its interior. Let $S^{2} \times E^{2}$ be a neighborhood of $S^{2}$ in $S^{4}$ with smooth boundary, so that $S^{4}-\left(S^{2} \times B^{2}\right)=A^{4}$ is an exterior of $S^{2}$. What other two-spheres in $S^{4}$ have an exterior homeomorphic to $A^{4}$ ? Joining the boundary $S^{2} \times S^{1}$ of the

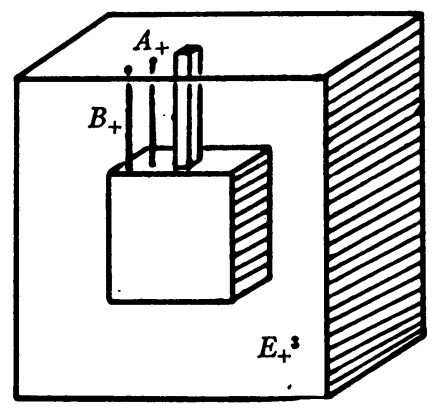

$5 a$

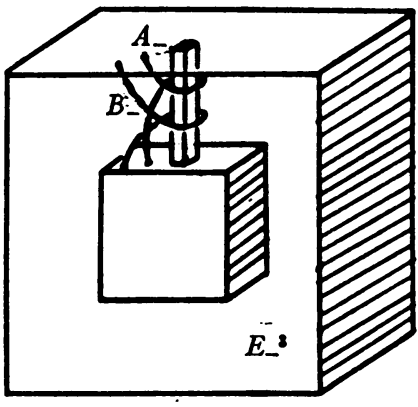

$5 \mathrm{~b}$

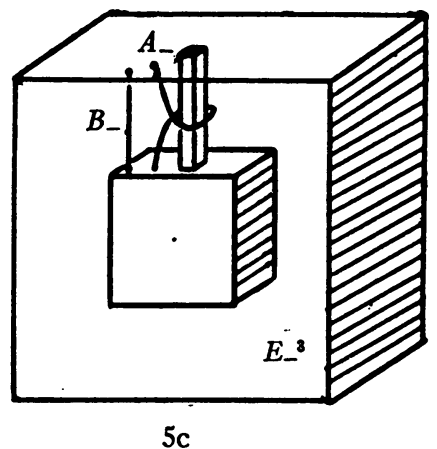

$5 c$

FIGURE 5

neighborhood $S^{2} \times E^{2}$ of $S^{2}$ to the boundary $S^{2} \times S^{1}$ of $A^{4}$, we obtain a fourmanifold $M^{4}$ and a distinguished smooth two-sphere in $M^{4}$. Every smooth embedding of a two-sphere in a four-manifold with an exterior homeomorphic to $A^{4}$ may be obtained, up to homeomorphism, in this manner. By Theorem (2.1), the homeomorphism type of the pair obtained depends only on the isotopy class of the homeomorphism, $f$, used to join the two boundaries. Hence, by Theorem (5.1), there are at most eight nonequivalent smooth embeddings of a two-sphere in a four-manifold with a given exterior. A fortiori, there are at most eight nonequivalent smooth embeddings of $S^{2}$ in $S^{4}$ with a given exterior. In this section we will cut this number down to two.

We are thus given $S^{2} \times E^{2}$ with boundary $S^{2} \times S^{1}$, and $A^{4}$ with boundary $S^{2} \times S^{1}$. Identify the two boundaries (ideally, not physically) so that we may speak of a homeomorphism connecting them as a homeomorphism of $S^{2} \times S^{1}$ 
onto itself. If $A^{4}$ was obtained as the exterior of some given two-sphere in the four-sphere, then there is a natural way of doing this. The particular identification, however, is unimportant, and is done only for the convenience it affords us in the verbal description of our constructions. If $f$ is a homeomorphism of $S^{2} \times S^{1}$ onto itself, let $[f]$ denote the isotopy class of $f$ in the group of homeomorphisms of $S^{2} \times S^{1}$. Thus $[f]$ is an element of $\mathcal{H}\left(S^{2} \times S^{1}\right)$.

Lemma (15.1). Let the pair $\left(M^{4}, S^{2}\right)$ be obtained by joining $S^{2} \times E^{2}$ to $A^{4}$ by the homeomorphism $f$ of $S^{2} \times S^{1}$. Similarly, let the pair $\left(N^{4}, S^{2}\right)$ be obtained by joining $S^{2} \times E^{2}$ to $A^{4}$ by the homeomorphism $g$ of $S^{2} \times S^{1}$. If $\left[g^{-1}\right][f] \in \mathcal{H C}^{\prime}$, then $\left(M^{4}, S^{2}\right)$ is homeomorphic to $\left(N^{4}, S^{2}\right)$.

Refer to $\$ 5$ for notation. Note that the homeomorphisms $(1,1),(1, s)$, $(r, 1)$ and $(r, s)$ of $S^{2} \times S^{1}$ extend to homeomorphisms of $\left(S^{2} \times E^{2}, S^{2} \times\right.$ origin $)$ onto itself. By Theorem (2.1), if $[h] \in \mathcal{H C}^{\prime}$, then the homeomorphism $h$ of $S^{2} \times S^{1}$ also extends to a homeomorphism of $\left(S^{2} \times E^{2}, S^{2} \times\right.$ origin). Suppose now that $\left[g^{-1}\right][f]=\left[g^{-1} f\right]$ is an element of $\mathfrak{F C}^{\prime}$. We construct a homeomorphism $h$ of $\left(M^{4}, S^{2}\right)$ onto $\left(N^{4}, S^{2}\right)$ as follows. On $A^{4}$, let $h$ be the identity. On $S^{2} \times S^{1}$ viewed as the boundary of $A^{4}, h$ is still the identity. But on $S^{2} \times S^{1}$ viewed as the boundary of $S^{2} \times E^{2}, h=g^{-1} f$. By the above remarks, $g^{-1} f$ extends to a homeomorphism $h$ of $\left(S^{2} \times E^{2}, S^{2} \times\right.$ origin), which we take as the definition of $h$ on $S^{2} \times E^{2}$. Thus $h$ is a homeomorphism of $\left(M^{4}, S^{2}\right)$ onto $\left(N^{4}, S^{2}\right)$.

Corollary. Let $S^{2}$ be a smooth two-sphere in $S^{4}$ with exterior $A^{4}$. Let $\left(M^{4}, S^{2}\right)$ be obtained by joining $S^{2} \times E^{2}$ to $A^{4}$ by the homeomorphism $f$ of $S^{2} \times S^{1}$. If $[f] \in \mathcal{H C}^{\prime}$, then $\left(M^{4}, S^{2}\right)$ is homeomorphic to the original pair $\left(S^{4}, S^{2}\right)$.

THEOREM (15.2). There are at most two nonequivalent embeddings of a twosphere in the four-sphere with a given exterior.

The following lemma shows that the simple technique of Lemma (15.1) is incapable of cutting down the number of possibly nonequivalent embeddings of $S^{2}$ in $S^{4}$ with a given exterior from two to one.

Lemma (15.3). The homeomorphism $T$ of $S^{2} \times S^{1}$ onto itself does not extend to a continuous map of $S^{2} \times E^{2}$ onto itself.

Suppose $T$ extends to the map $T^{\prime}$ of $S^{2} \times E^{2}$ onto itself. Then $p_{1} T$ extends to the map $p_{1} T^{\prime}$ of $S^{2} \times E^{2}$ onto $S^{2}$. Identify $S^{2}$ with $S^{2} \times$ origin, so that $p_{1}$ may be identified with the projection of $S^{2} \times E^{2}$ onto $S^{2} \times$ origin. Shrinking $S^{2} \times S^{1}$ over $S^{2} \times E^{2}$ to $S^{2} \times$ origin yields a homotopy of $p_{1} T$ with $p_{1} T^{\prime} p_{1}$. Since the induced mappings $p_{1}^{*}$ and $\left(p_{1} T\right)^{*}$ of $H^{2}\left(S^{2} ; Z\right)$ onto $H^{2}\left(S^{2} \times S^{1} ; Z\right)$ coincide, $p_{1} T^{\prime} / S^{2} \times$ origin must have degree +1 . Hence $p_{1} T^{\prime} p_{1}$ is homotopic to $p_{1}$. Thus $p_{1} T$ is homotopic to $p_{1}$, contradicting the results of $\S 14$. Hence no such extension $T^{\prime}$ exists, and the lemma is proved. 
If Lemma (15.1) is to be strengthened by replacing $\mathcal{H}^{\prime}$ by $\mathfrak{F}$, then the above lemma shows, in effect, that the proof must depend upon an analysis of the structure of $A^{4}$.

Again let $S^{2}$ be a smooth two-sphere in $S^{4}$ with an exterior $A^{4}$. Join the boundary $S^{2} \times S^{1}$ of $S^{2} \times E^{2}$ to the boundary $S^{2} \times S^{1}$ of $A^{4}$ by the homeomorphism $T$. We obtain a manifold $M^{4}$ with a distinguished smooth two-sphere, $S^{2} \times$ origin, which has an exterior homeomorphic to $A^{4}$. Theorem (5.1) and Lemma (15.1) imply that the original pair $\left(S^{4}, S^{2}\right)$ and the new pair $\left(M^{4}, S^{2}\right)$ give the only possible smooth embeddings of a two-sphere in a four-manifold with an exterior homeomorphic to $A^{4}$. Two questions now arise. Is $M^{4}$ a foursphere? If so, is $\left(M^{4}, S^{2}\right)$ homeomorphic to $\left(S^{4}, S^{2}\right)$ ? Until stated to the contrary, $M^{4}$ will have the meaning given above.

16. $M^{4}$ is a homotopy four-sphere. $T$ induces the identity automorphism of $\pi_{1}\left(S^{2} \times S^{1}\right)$, since $T / S^{2} \bigvee S^{1}$ is the identity. Thus, by the van Kampen theorem $[18 ; 19], M^{4}$ has the same fundamental group as $S^{4}$, i.e., $M^{4}$ is simply connected.

LeMma (16.1). $H_{2}\left(M^{4} ; Z\right)=0$.

Consider the following part of the homology sequence of the pair $\left(M^{4}, A^{4}\right)$.

$$
H_{2}\left(A^{4}\right) \stackrel{i}{\rightarrow} H_{2}\left(M^{4}\right) \stackrel{j}{\rightarrow} H_{2}\left(M^{4}, A^{4}\right) \stackrel{\partial}{\rightarrow} H_{1}\left(A^{4}\right) .
$$

By Alexander duality, $H_{2}\left(A^{4}\right)=H_{2}\left(\right.$ Int $\left.A^{4}\right)=H_{1}\left(S^{2} \times E^{2}\right)=0$, and $H_{1}\left(A^{4}\right)$ $=H_{1}\left(\operatorname{Int} A^{4}\right)=H_{2}\left(S^{2} \times E^{2}\right)=Z$. By excision, $H_{2}\left(M^{4}, A^{4}\right)=H_{2}\left(S^{2} \times E^{2}, S^{2} \times S^{1}\right)$ $=Z$. A generator of $H_{2}\left(M^{4}, A^{4}\right)$ is carried by $x_{n} \times E^{2}$. Under $\partial$, this generator goes into the generator of $H_{1}\left(A^{4}\right)$ represented by $x_{n} \in S^{1}$. Hence $\partial$ is an isomorphism, and thus $j$ is the zero homomorphism. But $i$ is also the zero homomorphism, since $H_{2}\left(A^{4}\right)=0$. Then by exactness, $H_{2}\left(M^{4}\right)=0$.

Since $M^{4}$ is orientable, $H_{3}\left(M^{4}\right)=H_{1}\left(M^{4}\right)=0, H_{4}\left(M^{4}\right)=Z$, and $H_{n}\left(M^{4}\right)=0$ for $n>4$. Thus $M^{4}$ is a simply connected four-dimensional manifold with the homology groups of the four-sphere.

We construct a mapping $f$ of $M^{4}$ onto $S^{4}$ as follows. Choose a four-simplex of $M^{4}$, and collapse the complement in $M^{4}$ of its interior to a point. Map the resulting four-sphere homeomorphically onto $S^{4}$. The induced mapping, $f_{*}: H_{4}\left(M^{4} ; Z\right) \rightarrow H_{4}\left(S^{4} ; Z\right)$ is clearly an isomorphism. That $f$ is then a homotopy equivalence follows from the next lemma, proved as corollary to Theorem 3 of [20].

Lemma (16.2). Let $X$ and $Y$ be connected compacta which are absolute neighborhood retracts. If $X$ and $Y$ are simply connected and $f: X \rightarrow Y$ induces isomorphisms $f_{*}: H_{n}(X) \rightarrow H_{n}(Y)$ for all $n \geqq 2$, then $f$ is a homotopy equivalence.

Hence $M^{4}$ is a homotopy four-sphere. If the generalized Poincaré conjecture is true in dimension four, then $M^{4}$ is homeomorphic to the four-sphere. However, to prove directly that $M^{4}$ is homeomorphic to the four-sphere necessitates an analysis of $A^{4}$. One way to obtain this result is to show that 
the homeomorphism $T$ of $S^{2} \times S^{1}$, considered as the boundary of $A^{4}$, is always extendable to a homeomorphism of $A^{4}$. Using the construction of Lemma (15.1) this would not only show that $M^{4}$ is a four-sphere, but would also show that $\left(M^{4}, S^{2}\right)$ is homeomorphic to $\left(S^{4}, S^{2}\right)$. We turn, therefore, to the problem of extending $T$ to a homeomorphism of $A^{4}$.

17. Spinning manifolds. Let $\Phi_{t}$ be a rotation of $S^{2}$ about the axis through the north and south poles through an angle $2 \pi t$ in a fixed direction. Then $\left\{\Phi_{t}\right\}, t \in[0,1]$, is a one-parameter family of homeomorphisms of $S^{2}$ beginning and ending at the identity. Let $M^{3}$ be a compact three-manifold with the single boundary component $S^{2}$. If $\left\{\Phi_{t}\right\}$ can be extended to a one-parameter family of homeomorphisms $\left\{\bar{\Phi}_{t}\right\}$ of $M^{3}$, beginning and ending at the identity, then $\left\{\bar{\Phi}_{t}\right\}$ is said to determine a spinning of $M^{3}$, and we say that $M^{3}$ can be spun. It is easily shown that this definition is independent of the way we identify our unit two-sphere with the two-sphere boundary of $M^{3}$.

Consider the two-sphere $S^{2} \times 0$ on the boundary $S^{2} \times S^{1}$ of $A^{4}$. Suppose that $S^{2} \times 0$ is the boundary of a three-manifold $M^{3}$ in $A^{4}$ whose intersection with $S^{2} \times S^{1}$ is precisely $S^{2} \times 0$. We say that $M^{3}$ is smoothly embedded in $A^{4}$ if $M^{3}$ has a neighborhood $M^{3} \times I$ in $A^{4}$ whose intersection with $S^{2} \times S^{1}$ is a neighborhood $S^{2} \times I$ of $S^{2} \times 0$ on $S^{2} \times S^{1}$. Suppose now that $S^{2} \times 0$ bounds a smooth $M^{3}$ in $A^{4}$. Then using Theorem (3.1), it is easy to show that $M^{3}$ has a neighborhood $M^{3} \times I$ in $A^{4}$ whose intersection with $S^{2} \times S^{1}$ is $S^{2} \times[-1 / 4,1 / 4]$.

TheOREM (17.1). Let $S^{2}$ be a smooth two-sphere in $S^{4}$ with exterior $A^{4}$. Suppose that $S^{2} \times 0$ is the boundary of an orientable three-manifold $M^{3}$, smoothly embedded in $A^{4}$. If $M^{3}$ can be spun, then the homeomorphism $T$ of $S^{2} \times S^{1}$ extends to a homeomorphism of $A^{4}$.

Recalling the definition of $\Phi_{t}$ given at the beginning of this section, $T$ is defined by $T(x, t)=\left(\Phi_{t}(x), t\right) . T$ is clearly isotopic to the homeomorphism $T^{*}$ defined by

$$
\begin{aligned}
T^{*}(x, t) & =\left(\Phi_{2 t+1 / 2}(x), t\right), & \text { for } 0 \leqq t \leqq 1 / 4 \text { and } \text { for } 3 / 4 \leqq t \leqq 1 ; \\
T^{*}(x, t) & =(x, t), & \text { for } 1 / 4 \leqq t \leqq 3 / 4 .
\end{aligned}
$$

$T^{*}$ simply concentrates the rotational action of $T$ between $t=3 / 4(=-1 / 4)$ and $t=1 / 4$. By Theorem (2.1), it is sufficient to extend $T^{*}$ to a homeomorphism of $A^{4}$. By assumption, $M^{3}$ has a neighborhood in $A^{4}$ of the form $M^{3} \times[-1 / 4,1 / 4]$ whose intersection with $S^{2} \times S^{1}$ is $S^{2} \times[-1 / 4,1 / 4]$. Since $M^{3}$ can be spun, the one-parameter family of homeomorphisms $\left\{\Phi_{t}\right\}$ of $S^{2}$ extends to a one-parameter family of homeomorphisms $\left\{\bar{\Phi}_{t}\right\}$ of $M^{3}$, beginning and ending at the identity. We can thus extend $T^{*}$ on $S^{2} \times S^{1}$ to $T^{*}$ on $\left(S^{2} \times S^{1}\right) \cup\left(M^{3} \times[-1 / 4,1 / 4]\right)$ by defining

$$
T^{*}(m, t)=\left(\bar{\Phi}_{2 t+1 / 2}(m), t\right), \quad \text { for } m \in M^{3} \text { and }-1 / 4 \leqq t \leqq 1 / 4 .
$$

Look now at the open set $U$ in $A^{4}$ on which $T^{*}$ has not yet been defined. On the boundary of $U, T^{*}$ has been defined as the identity. Thus extend $T^{*}$ 
over $U$ via the identity, which completes the extension of $T^{*}$ over $A^{4}$ and proves the theorem.

CoRollary. Under the same conditions stated in Theorem (17.1), $\left(M^{4}, S^{2}\right)$ is homeomorphic to $\left(S^{4}, S^{2}\right)$, and hence there is, up to homeomorphism, just one smooth embedding of $S^{2}$ in a four-manifold with an exterior homeomorphic to $A^{4}$.

By Theorem (17.1), $T$ extends to a homeomorphism $T$ of $A^{4}$. Define a homeomorphism $h$ of $\left(S^{4}, S^{2}\right)$ onto $\left(M^{4}, S^{2}\right)$ as follows. On $A^{4}$, let $h=T$. Thus on $S^{2} \times S^{1}$ viewed as the boundary of $A^{4}, h=T$. Hence on $S^{2} \times S^{1}$ viewed as the boundary of $S^{2} \times E^{2}, h$ is the identity. Then extend $h$, via the identity, over $S^{2} \times E^{2}$, and the corollary is proved.

18. Restriction to the semi-linear case. From this point on we shall assume that $S^{2}$ is a smoothly embedded, semi-linear two-sphere in the foursphere $S^{4}$ (see $\$ 1$ for definitions).

Suppose that $S^{2}$ is a semi-linear two-sphere in $S^{4}$. If $v$ is a vertex of $S^{2}$, then the link of $v$ on $S^{2}$ is a simple closed curve, while the link of $v$ in $S^{4}$ is a three-sphere. If this simple closed curve is unknotted in this three-sphere, then $S^{2}$ is said to be locally flat at $v$. If $S^{2}$ is locally flat at all of its vertices, then $S^{2}$ is said to be locally flat. Every smoothly embedded semi-linear two-sphere in $S^{4}$ is clearly locally flat. Conversely $\left(^{3}\right)$, every locally flat semi-linear twosphere in $S^{4}$ is smoothly embedded, and its regular neighborhoods are homeomorphic to $S^{2} \times E^{2}$.

Let $S^{2}$ be a locally flat semi-linear two-sphere in $S^{4}$. Suppose it is known that $S^{2}$ bounds an orientable semi-linear three-manifold $M^{3}$ in $S^{4}$. $A^{4}$ is the complement in $S^{4}$ of an open regular neighborhood of $S^{2}$. Then it is clear that $A^{4} \cap M^{3}$ is a three-manifold whose boundary two-sphere lies on the boundary of $A^{4} . M^{3}$ may also meet the boundary of $A^{4}$ elsewhere, but these intersections can be pushed off the boundary. Once this is done, the deformed $A^{4} \cap M^{3}$ is smoothly embedded in $A^{4}$ by [22]. Furthermore, the boundary of $A^{4} \cap M^{3}$ can be taken as $S^{2} \times 0$ on a parametrization of the boundary of $A^{4}$ as $S^{2} \times S^{1}$. Finally, $A^{4} \cap M^{3}$ is easily seen to be homeomorphic to $M^{3}$. Hence in order to satisfy all the hypotheses of Theorem (17.1), it would have to be shown that $M^{3}$ could be spun.

A first step will be to show that every locally flat semi-linear two-sphere in $S^{4}$ is the boundary of an orientable semi-linear three-manifold in $S^{4}$.

\section{THE EMBEDDING OF ORIENTABLE SURFACES IN FOUR-SPACE}

19. Preliminary remarks. Let $M^{2}$ be a locally flat, polyhedral, closed orientable surface (not necessarily connected) in Euclidean four-space $R^{4}$. In this chapter we will outline a proof that $M^{2}$ is the boundary of an orientable polyhedral three-manifold in $R^{4}$. The arguments may be found in greater detail in the author's thesis.

(8) The proof given in [21] is incorrect. A new proof will appear shortly. 
$M^{2}$ is first deformed slightly so that its intersections with the horizontal hyperplanes $R_{t}^{3}=\left\{\left(x_{1}, x_{2}, x_{3}, x_{4}\right): x_{4}=t\right\}$ are as simple as possible. In particular we arrange that no two vertices of $M^{2}$ have the same $i$ th coordinate for $i=1,2,3$ or 4 , and that no two-simplex of $M^{2}$ lies in a plane parallel to the $x_{4}$-axis. If $R_{t}^{3}$ contains the vertex $v$ of $M^{2}$, then, since no other vertex of $M^{2}$ lies in $R_{t}^{3}$, the number of points on the link of $v$ in $M^{2}$ with fourth coordinate $t$ is finite and even. It is arranged, by slight deformation of $M^{2}$, that this number, $n$, assumes only the values 0,2 and 4 .

Now as $t$ decreases, $M^{2} \cap R_{t}^{3}$ changes isotopically, except at the finite number of singular values of $t$ for which $R_{t}^{3}$ contains a vertex of $M^{2}$. If $n=0$ at a vertex $v$ of $M^{2}$, then as $t$ decreases through the fourth coordinate of this vertex, $M^{2} \cap R_{t}^{3}$ changes isotopically, except that an unknotted simple closed curve shrinks to a point and then disappears (or vice versa). This will be called an elliptic transformation. If $n=2$ at $v$, then as $t$ decreases through the fourth coordinate of $v, M^{2} \cap R_{\imath}^{3}$ changes isotopically. If $n=4$ at $v$, then as $t$ decreases through the fourth coordinate of $v, M^{2} \cap R_{t}^{3}$ changes isotopically, except that two arcs come together at their midpoints and then separate like the crosssections of a saddle surface. This will be called a hyperbolic transformation.
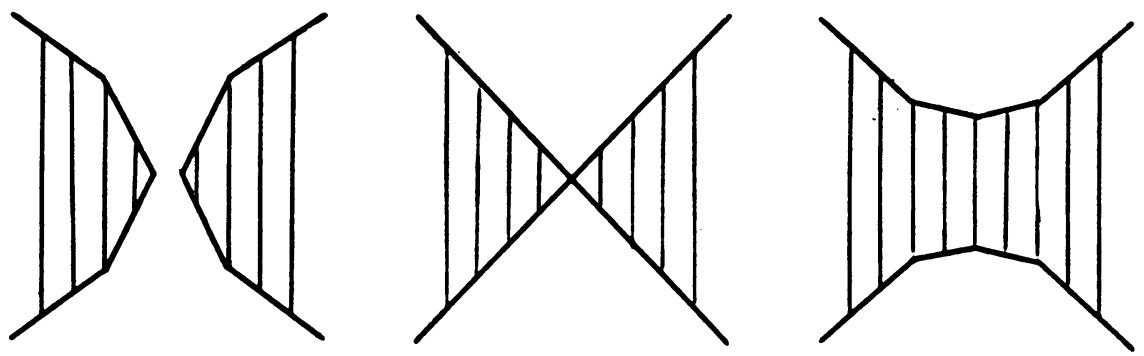

FIGURE 6

20. The construction of $M^{3}$. What we have in mind is to find orientable surfaces in the $R_{t}^{3}$ whose boundaries are precisely $M^{2} \cap R_{t}^{3}$, in such a continuous way that when considered together they form an orientable three-manifold $M^{3}$ whose boundary is $M^{2}$. The selection of these orientable surfaces, $M^{3} \cap R_{t}^{3}$, is carried out with decreasing $t$, and is begun, as $M^{2} \cap R_{t}^{3}$ opens up from a point into an unknotted simple closed curve, by having $M^{3} \cap R_{t}^{3}$ open up from a point into a two-cell. If $M^{2} \cap R_{t}^{3}$ changes isotopically, $M^{3} \cap R_{t}^{3}$ can be dragged along isotopically. It remains to decide what to do with $M^{3} \cap R_{t}^{3}$ when $M^{2} \cap R_{t}^{3}$ undergoes hyperbolic and elliptic transformations.

In the hyperbolic case, the arcs which come together already form part of the boundary of a cross-sectional surface and the hyperbolic transformation could be extended to the surface as shown in Figure 6, except for the possibility that a number of sheets of the surface may be in the way. These sheets are simply pierced one after the other with decreasing $t$, the cuts being 
joined as in Figure 7 to preserve orientability of the cross-sections. Finally, when no more sheets are in the way, the original hyperbolic transformation is extended to the cross-sectional surface. That this final transformation does not destroy the orientability of the cross-sections is shown in $\$ 21$.

In the case of the elliptic transformations, if with decreasing $t$ a point opens up into an unknotted simple closed curve, we simply introduce another
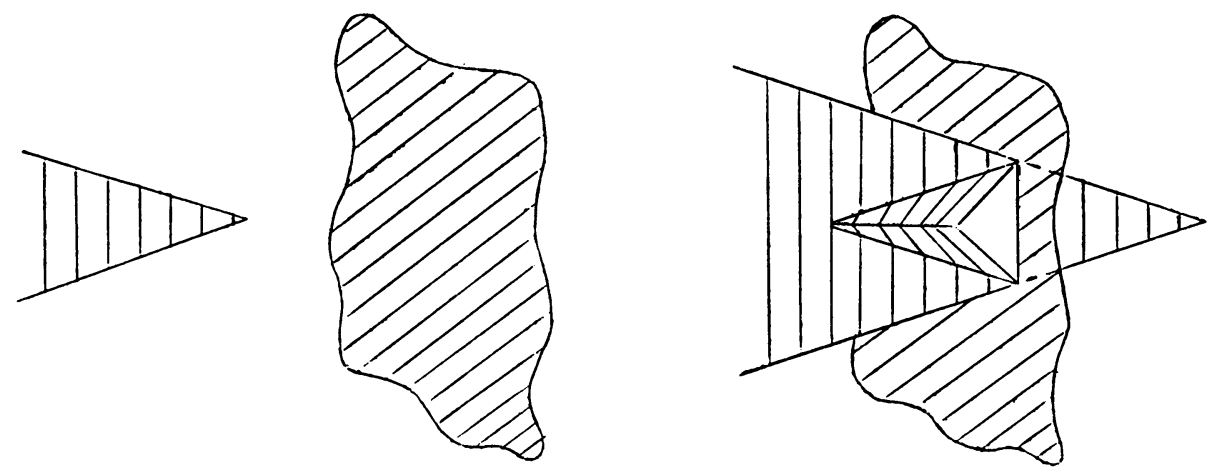

Figure 7

component of the cross-sectional surface which with decreasing $t$ opens up from a point into a two-cell. The serious case occurs when a component of $M^{2} \cap R_{t}^{3}$ shrinks to a point and then disappears with decreasing $t$. Call this component $c_{1}$, and let $c_{2}, \cdots, c_{k}$ be the other boundary curves of the component $G$ of the cross-sectional surface containing $c_{1}$. Since $c_{1}$ is unknotted by the local flatness of $M^{2}$, let $D$ be a polyhedral two-cell in $R_{t}^{3}$ bounded by $c_{1}$.
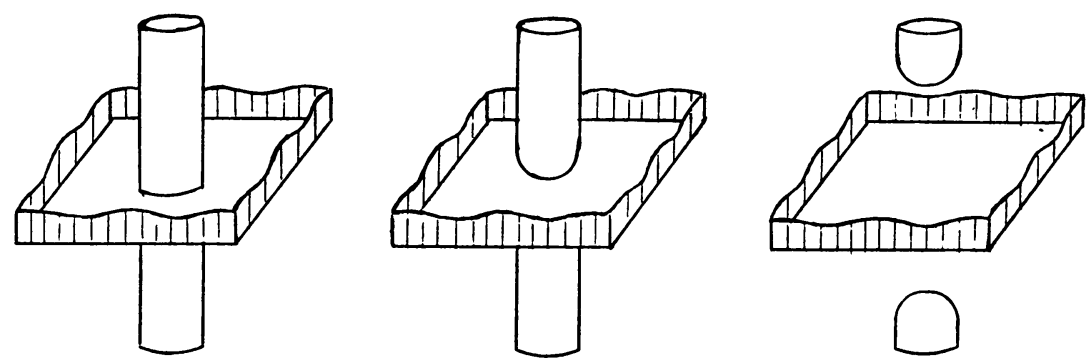

FIGURE 8

Let $c^{\prime}$ be a simple closed curve on $G$ lying in a small neighborhood of $c_{1}$ and "parallel" to $c_{1}$. Because the cross-sectional surface, and hence $G$, is orientable, the linking number of $c^{\prime}$ with $c_{1}$ is the same as the sum of the linking numbers of the $c_{2}, \cdots, c_{k}$ with $c_{1}$. But each of these linking numbers is zero, since $c_{1}$ is about to shrink to a point away from all these curves. Because the 
linking number of $c^{\prime}$ with $c_{1}$ is zero, the cross-sectional surface can be deformed so that a small neighborhood of $c_{1}$ on $G$ meets $D$ only at $c_{1}$, while the total intersection of the cross-sectional surface with $D$ consists of a number of simple closed curves. Each of these intersections can be removed by standard hyperbolic transformations with decreasing $t$, as shown in Figure 8, until finally $D$ meets the cross-sectional surface only at its boundary curve $c_{1}$. By a slight deformation of $M^{2}$, the original elliptic transformation can be altered so as to shrink $c_{1}$ to a point along $D$, closing up a component of the crosssectional surface and completing the construction for the elliptic transformation.

When finally $t$ has decreased below the minimum value attained by the fourth coordinates of points of $M^{2}$, the cross-sectional surface consists of a number of closed orientable surfaces in a three-dimensional hyperplane $R_{t}^{3}$. It remains to shrink off the components of this surface to points with decreasing $t$. If the resulting $M^{3}$ is to be a manifold, this must be done by first changing these components into two-spheres. But R. H. Fox has shown in [25, Theorem 2] that whenever we are given a number of polyhedral closed orientable surfaces in three-space, not all of which are two-spheres, a hyperbolic transformation may be found which either decreases the total genus or else increases the number of components with positive genus while leaving the total genus unaltered. We carry out such a transformation with decreasing $t$, and repeat the procedure until all the components of the cross-sectional surface are two-spheres, which may then be shrunk to points as $t$ decreases further, completing the construction of $M^{3}$.

R. H. Bing has pointed out to me that if we are willing to allow a threedimensional cross-section, then the argument can be completed as soon as the cross-sectional surface becomes closed, for every closed surface in threespace, whether connected or not, is certainly the boundary of a three-dimensional region.

As the various transformations undergone by the cross-sectional surfaces are topologically equivalent to those experienced by a cross-section of a hypersurface in $R^{4}$ (with due regard being taken of the fact that $M^{3}$ has a boundary), it is easily seen that $M^{3}$ is a manifold. Since the cross-sections are orientable and the various transformations preserve orientations, $M^{3}$ is also orientable.

It remains to be seen that the final transformation in the hyperbolic case does not destroy the orientability of the cross-sections.

21. The orientability of the cross-sectional surfaces. Let $c_{1}, c_{2}, \cdots, c_{k}$ be a family of disjoint oriented simple closed curves on an orientable twomanifold $M^{2}$. The family will be said to be oriented consistently if for each component $D_{j}$ of $M^{2}-\left(\cup_{i} c_{i}\right)$, the orientation of the boundary of $D_{j}$ is induced by one of the two possible orientations of $D_{j}$.

By a nonsingular value of $t$ we shall now mean a value of $t$ for which the 
cross-sectional surface $M^{3} \cap R_{t}^{3}$ is a manifold without singularity. We will show that $M^{3} \cap R_{t}^{3}$ can be oriented in such a way as to make $M^{2} \cap R_{t}^{3}$, with orientations induced by $M^{3} \cap R_{t}^{3}$, a consistently oriented family of simple closed curves on $M^{2}$. In particular, the cross-sectional surfaces will be orientable. If $M^{3} \cap R_{t}^{3}$ is oriented so that $M^{2} \cap R_{t}^{3}$ is consistently oriented with respect to $M^{2}$, then we refer to $M^{3} \cap R_{t}^{3}$ as being consistently oriented (with respect to $M^{2}$ ).

If $t$ is in the first interval of nonsingular values, $M^{2} \cap R_{t}^{3}$ is a simple closed curve on $M^{2}$ which has expanded from a point, and therefore separates $M^{2}$. Since $M^{2}$ is orientable, each of the residual components of this curve can be oriented so as to induce a given orientation on the curve. This orientation then gives an orientation for $M^{3} \cap R_{t}^{3}$, so that at the beginning the cross-sectional surface can be consistently oriented. We must show that the various transformations to which the cross-sectional surface is subjected with decreasing $t$ do not destroy this property.

In an elliptic transformation in which a curve $c$ expands from a point, one of the domains of $M^{2}-c$ contains no other curves, and hence $c$ may be oriented so that $M^{2} \cap R_{t}^{3}$ is still consistently oriented. This orientation of $c$ gives an orientation for the new two-cell bounded by $c$ in $M^{3} \cap R_{t}^{3}$, and hence $M^{3} \cap R_{t}^{3}$ is still consistently oriented. If in the elliptic transformation a curve $c$ shrinks to a point, then again one of the domains of $M^{2}-c$ contains no other curves. Hence the remaining curves are still consistently oriented, and $M^{3} \cap R_{t}^{3}$ remains consistently oriented as one of its components loses a boundary curve.

Suppose now that we have a hyperbolic transformation during which curves $c$ and $c^{\prime}$ come together at a point (the case $c=c^{\prime}$ is not excluded). For such a transformation to occur, it is necessary that $c$ and $c^{\prime}$ appear together in the boundary of some domain of $M^{2}-\left(M^{2} \cap R_{t}^{3}\right)$. Since this domain is orientable, $c$ and $c^{\prime}$ can only come together with their orientations "locally opposite." Then the new cross-sectional surface is still orientable, and its orientation is induced from that of the old cross-sectional surface in the obvious way. To see that the new $M^{3} \cap R_{t}^{3}$ is consistently oriented, we have only to observe the hyperbolic transformation undergone by $M^{2} \cap R_{t}^{3}$ on $M^{2}$. Locally, one domain of $M^{2}-\left(M^{2} \cap R_{\imath}^{3}\right)$ is split, while two others are joined. The new domains then receive orientations in a natural way from the old, and these new orientations demonstrate the consistent orientation of the new $M^{2} \cap R_{t}^{3}$. Since the orientation of the new $M^{2} \cap R_{t}^{3}$ is also induced by the orientation of the new $M^{3} \cap R_{t}^{3}$, the new $M^{3} \cap R_{t}^{3}$ is consistently oriented.

The auxiliary transformations used in $\$ 20$ do not affect the consistent orientation of the cross-sectional surfaces, and hence we have shown that the cross-sectional surfaces may be consistently oriented, and that the consistent orientation behaves continuously under the various transformations.

We now have 
THEOREM (21.1). Every locally flat polyhedral closed orientable surface in Euclidean four-space is the boundary of an orientable polyhedral three-manifold in four-space.

22. Two-spheres in the four-sphere which are uniquely determined by their exteriors. Let $S^{2}$ be a locally flat semi-linear two-sphere in $S^{4}$. By Theorem (21.1), $S^{2}$ is the boundary of an orientable semi-linear three-manifold $M^{3}$ in $S^{4}$. $\$ 18$ and the corollary to Theorem (17.1) imply that if $M^{3}$ can be spun, then any two-sphere in $S^{4}$ whose exterior is homeomorphic to the exterior of $S^{2}$ is actually equivalent to $S^{2}$. Recall that in the semi-linear case, the exterior is a uniquely defined semi-linear four-manifold. If every locally flat semi-linear two-sphere in $S^{4}$ were the boundary of a three-manifold in $S^{4}$ which could be spun, then the exterior of $S^{2}$ would be a complete invariant for the embedding in the semi-linear case $\left({ }^{4}\right)$. While we are unable to show this, it is at least clear that some two-spheres in $S^{4}$ bound three-manifolds which can be spun. For example, if $\left(S^{4}, S^{2}\right)$ is obtained by spinning a knot in threespace (see $[27 ; 28]$ ), then an orientable surface bounded by the knot spins into a three-manifold bounded by $S^{2}$. But the very method of generation of this three-manifold shows that it can be spun in the sense of $\$ 17$. Noting that a two-sphere obtained by spinning a tame knot is locally flat, we have the following result.

THEOREM (22.1). Every semi-linear two-sphere in the four-sphere obtained by spinning a tame knot is uniquely determined by its semi-linear exterior.

\section{The EMBEDding of $N$-SPHERES IN THE $N+2$ SPHERE}

23. $N>2$. In this brief chapter we consider the possibility of obtaining results for $n \neq 2$ similar to those obtained for $n=2$. The problem divides naturally into the case $n>2$ and the case $n=1$.

When $n>2$, we are faced with the problem of determining the group of homeomorphism types of $S^{n} \times S^{1}$. Unfortunately, most isotopy problems for manifolds of dimension greater than three are as yet unsolved. The best we can say is that if $G$ is the group of homeomorphisms of $S^{n} \times S^{1}$ and $G_{0}^{\prime}$ the normal subgroup consisting of those homeomorphisms homotopic to the identity, then $G / G_{0}^{\prime}=Z_{2}+Z_{2}+Z_{2}$. The proof is simple, but depends heavily upon the classification of maps of an $n+1$ complex into the $n$-sphere given by Steenrod [29]. If two homeomorphisms of $S^{n} \times S^{1}$ are isotopic if and only if they are homotopic, then $G / G_{0}=G / G_{0}^{\prime}$, and we immediately obtain the result that there are at most two non-equivalent smooth embeddings of an $n$-sphere in the $n+2$ sphere with a given exterior. While we are unable to prove that every locally flat polyhedral $n$-sphere in $n+2$ space is the boundary of a poly-

(4) Those three-manifolds which san be spun (in a somewhat more restrictive sense) have been characterized in [31]. 
hedral $n+1$ manifold, we can at least obtain a result about spun $n$-spheres similar to Theorem (22.1).

24. $N=1$. Here the problem is entirely different, for the group of homeomorphism types of $S^{1} \times S^{1}$ is no longer finite, but is the two-dimensional unimodular group. Furthermore, when a neighborhood of $S^{1}$ is adjoined to its exterior by a homeomorphism other than the identity, the resulting threemanifold may not be the three-sphere. It seems, at least in simple cases, that whenever one gets the three-sphere, the new knot is equivalent to the original one. Partial results are easily obtained, however, and we state without proof the following theorem.

THEOREM (24.1). Let $k$ and $k^{\prime}$ be tame knots in $S^{3}$ such that $S^{3}-k$ is homeomorphic to $S^{3}-k^{\prime}$. If $k$ is a torus knot, then $k$ and $k^{\prime}$ are equivalent.

\section{REFERENCES}

1. J. H. C. Whitehead, On doubled knots, J. London Math. Soc. 12 (1937), 63-71.

2. R. H. Fox and E. Artin, Some wild cells and spheres in three-dimensional space, Ann. of Math. (2) 49 (1948), 979-990.

3. M. Brown, Locally flat imbeddings of topological manifolds, Ann. of Math. (2) 75 (1962), 331-341.

4. J. H. C. Whitehead, Simplicial spaces, nuclei and m-groups, Proc. London Math. Soc. 45 (1938), 243-327.

5. M. Brown, A proof of the generalized Schoenfties theorem, Bull. Amer. Math. Soc. 66 (1960), 74-76.

6. J. W. Alexander, On the deformation of an n-cell, Proc. Nat. Acad. Sci. 9 (1923), 406-407.

7. T. Rado, Über den Begriff der Riemannschen Fläche, Acta-Litt. Sci. Szeged 2 (1925), 101121.

8. E. E. Moise, Affine structures in 3-manifolds. IV, Ann. of Math. (2) 56 (1952), 96-114.

9. R. H. Bing, An alternative proof that 3-manifolds can be triangulated, Ann. of Math. (2) 69 (1959), 37-65.

10. G. M. Fisher, On the group of all homeomorphisms of a manifold, Trans. Amer. Math. Soc. 97 (1960), 193-212.

11. G. S. McCarty, Jr., Homeotopy groups, Abstract 61T-48, Notices Amer. Math. Soc. 8 (1961), 67.

12. G. T. Whyburn, Topological analysis, Princeton Univ. Press, Princeton, N. J., 1958.

13. H. L. Smith, On continuous representations of a square upon itself, Ann. of Math. 19 (1918), 137-141.

14. R. H. Bing, Approximating surfaces with polyhedral ones, Ann. of Math. (2) 65 (1957), 456-483.

15. - Locally tame sets are tame, Ann. of Math. (2) 59 (1954), 145-158.

16. C. D. Papakyriakopoulos, On Dehn's lemma and the asphericity of knots, Ann. of Math. (2) 66 (1957), 1-26.

17. L. Pontrjagin, A classification of mappings of the three-dimensional complex into the twodimensional sphere, Recueil Mathématique 9 (1941), 331-359.

18. E. R. van Kampen, On the connection between the fundamental groups of related spaces, Amer. J. Math. 55 (1933), 261-267.

19. R. H. Crowell, On the van Kampen theorem, Pacific J. Math. 9 (1959), 43-50.

20. J. H. C. Whitehead, On the homotopy type of $A N R$ 's, Bull. Amer. Math. Soc. 54 (1948), 1133-1145. 
21. H. Noguchi, On regular neighborhoods of 2-manifolds in 4-Euclidean space. I, Osaka Math. J. 8 (1956), 225-242; Errata, Osaka Math. J. 9 (1957), 241-242.

22. - The thickening of combinatorial $n$-manifolds in $n+1$ space, Osaka Math. J. 12 (1960), 97-112.

23. H. Schubert, Die eindeutige Zerlegbarkeit eines Knotens in Primknoten, Sitz. Heidelberg. Akad. Wiss. Math. Nat. 3 (1949), 57-104.

24. B. Mazur, The definition of equivalence of combinatorial embeddings, Inst. Hautes Etudes Sci. Publ. Math. 3 (1959), 5-17. 470.

25. R. H. Fox, On the imbedding of polyhedra in 3-space, Ann. of Math. (2) 49 (1948), 462-

26. J. Milnor, A unique decomposition theorem for three-manifolds, Amer. J. Math. 84 (1962), 1-7.

27. E. Artin, Zur Isotopie Zweidimensionaler Flächen in $R_{\mathbb{A}}$, Abh. Math. Sem. Univ. Hamburg 4 (1925), 174-177.

28. J. J. Andrews and M. L. Curtis, Knotted 2-spheres in the 4-sphere, Ann. of Math. (2) 70 (1959), 565-571.

29. N. Steenrod, Products of cocycles and extensions of mappings, Ann. of Math (2) 48 (1947), 290-320.

30. M. H. A. Newman, On the superposition of $n$-dimensional manifolds, J. London Math. Soc. 2 (1927), 56-64.

31. H. Gluck, Tangled manifolds, Ann. of Math. (2) 75 (1962).

Princeton University,

Princeton, NeW Jersey 